\title{
THE RIEMANNIAN MEAN AND MATRIX INEQUALITIES RELATED TO THE ANDO-HIAI INEQUALITY AND CHAOTIC ORDER
}

\author{
TAKEAKI YAMAZAKI
}

Abstract. The Riemannian mean on the convex cone of positive definite matrices is a kind of geometric mean of $n$-matrices which is an extension of the geometric mean of two-matrices. In this paper, we derive the Ando-Hiai inequality for the Riemannian mean which is an extension of the well-known Ando-Hiai inequality of two-matrices. Moreover, we shall show an extension of a characterization of chaotic order. Lastly, we will give a negative answer for the problem whether the same results are satisfied or not for other geometric means of $n$-matrices.

Mathematics subject classification (2010): Primary 47A64; Secondary 47A63, 47L25.

Keywords and phrases: Positive definite matrices, the Riemannian manifold, the Riemannian mean, matrix inequality, operator inequality, arithmetic-geometric mean inequality, the Ando-Hiai inequality, chaotic order.

\section{REFERENCES}

[1] T. Ando, On some operator inequalities, Math. Ann. 279 (1987), 157-159.

[2] T. ANDO AND F. HIAI, Log majorization and complementary Golden-Thompson type inequalities, Linear Algebra Appl. 197, 198 (1994), 113-131.

[3] T. Ando, C. K. Li And R. Mathias, Geometric means, Linear Algebra Appl. 385 (2004), $305-334$.

[4] R. Bhatia, Positive definite matrices, Princeton Series in Applied Mathematics, Princeton University Press, Princeton, NJ, 2007.

[5] R. Bhatia AND J. Holbrook, Riemannian geometry and matrix geometric means, Linear Algebra Appl. 413 (2006), 594-618.

[6] R. Bhatia AND R. L. KARANDiKAR, Monotonicity of the matrix geometric mean, to appear in Math. Ann.

[7] D. A. Bini, B. MeIni AND F. Poloni, An effective matrix geometric mean satisfying the Ando-LiMathias properties, Math. Comp. 79 (2010), 437-452.

[8] M. FujiI, T. Furuta AND E. KAMEI, Furuta's inequality and its application to Ando's theorem, Linear Algebra Appl. 179 (1993), 161-169.

[9] T. FuRUTA, Applications of order preserving operator inequalities, Oper. Theory Adv. Appl. 59 (1992), 180-190.

[10] S. IzUmino And N. NAKAmura, Geometric means of positive operators II, Sci. Math. Jpn. 69 (2009), 35-44.

[11] J. D. LAWSON AND Y. Lim, Monotonic properties of the least squares mean, Math. Ann. 351 (2011), 267-269.

[12] H. LEE, Y. LIM AND T. YAMAZAKI, Multi-variable weighted geometric means of positive definite matrices, Linear Algebra Appl. 435 (2011), 307-322.

[13] M. MOAKHER, A differential geometric approach to the geometric mean of symmetric positive-definite matrices, SIAM J. Matrix Anal. Appl. 26 (2005), 735-747.

[14] R. D. Nussbaum And J. E. Cohen, The arithmetic-geometric mean and its generalizations for noncommuting linear operators, Ann. Scuola Norm. Sup. Pisa Cl. Sci. (4) 15 (1988), 239-308.

[15] K.-T. Sturm, Probability measures on metric spaces of nonpositive curvature, Heat kernels and analysis on manifolds, graphs, and metric spaces, 357-390, Contemp. Math. 338, Amer. Math. Soc., Providence, RI, 2003. 
[16] M. UChiYama, Some exponential operator inequalities, Math. Inequal. Appl. 2 (1999), 469-471.

[17] T. YAMAZAKI, An elementary proof of arithmetic-geometric mean inequality of the weighted Riemannian mean of positive definite matrices, preprint. 